\title{
Study of Communication Between Distributed Generation Devices in an Smart Grid Environment
}

\author{
J. A. Dias, P. J. A. Serni, A. M. Bueno, E. P. Godoy, Member, IEEE
}

\begin{abstract}
The increasing demand of electricity, due to population growth in recent years has resulted in a necessary expansion of the energy matrix through alternative sources including consumer's small power generators in low or medium voltage. Thus, studies have been conducted to enter the automation in the control of these systems but has not yet been established what will be controlled and how to get the data effectively. In this microgrid scenario, one of the challenges is the automation of network among different devices such as generator controllers, smart meters, etc., in order to facilitate the required exchange of information and enable networked control. Therefore, this paper presents a simulation of a microgrid $(M G)$ with a distributed generator $(D G)$ remotely controlled via a wireless communication. The methodology is presented through integrated simulation of smart grids, in which the electrical system was implemented using PLECS blockset, while the wireless network was designed using the TrueTime toolbox, both emulated in Matlab Simulink. In this MG application, the use of ZigBee and WLAN wireless networks to control the active power generation of the $D G$ is evaluated under different network parameters such as loss probability and distance between the devices. Results show that the simulation provide a solution for wireless network analysis, $D G$ control and electrical network modeling of the in smart $M G$ scenarios presenting the efficient network solution for MG development.
\end{abstract}

Keywords- Smart Microgrid, Distributed Generator, Integrated Simulation, TrueTime.

\section{INTRODUÇÃO}

$\mathrm{D}$ evido ao crescimento populacional nos últimos anos, em muitos países, a demanda pela utilização da energia elétrica resultou na adoção de políticas de incentivo à redução de gases causadores de efeito estufa através da diversificação das matrizes energéticas, por meio de fontes alternativas. Isso fez com que o uso de tecnologias de geração distribuída de baixa ou média potência, incluindo fontes de energia renováveis ou alternativas e elementos de armazenamento de energia, dessem origem às microredes [1]. Como cada unidade desse tipo possui um conversor de potência chaveado, originou-se então as "fontes de energia inteligentes" [2]. Essa é, portanto, a origem das redes inteligentes $(R I)$ ou do inglês Smart Grids, nas quais as fontes de energia auxiliam em atender a demanda de energia através da otimização de recursos de energia por meio de controle [3, 4].

Sendo assim, estudos vem sendo realizados para tornar o

J. A. Dias, Unesp - Univ. Estadual Paulista, Sorocaba, SP, Brasil. jeffdias2000@yahoo.com.br.

P. J. A. Serni, E. P. Godoy, Unesp - Univ. Estadual Paulista, Sorocaba, SP, Brasil. serni@sorocaba.unesp.br, epgodoy@sorocaba.unesp.br. controle desses sistemas automáticos. Entretanto, existe grande dificuldade em estabelecer o que será controlado e como obter os dados de forma eficaz [5].

Neste cenário de redes inteligentes, um dos grandes desafios corresponde ao processo de comunicação da rede entre os diferentes dispositivos, tais como controladores de geradores, medidores inteligentes, etc., a fim de facilitar a troca de informações necessárias e melhorar o desempenho da rede de comunicação.

Portanto, este trabalho apresenta um estudo da simulação de uma microrrede $(M R)$, com gerador distribuído $(G D)$, controlado remotamente através de uma rede de comunicação sem fio.

A metodologia é apresentada por meio da simulação integrada de redes inteligentes, na qual o sistema elétrico foi implementado através do software PLECS-blockset [6], enquanto que a rede sem fio foi parametrizada através do software TrueTime-toolbox [4], ambos simulados no ambiente Matlab-Simulink. Nesta aplicação de $M R$, o uso da rede de comunicação sem fio para controlar a geração da potência ativa do $G D$ é avaliada sob diferentes parâmetros de rede de comunicação, tais como a probabilidade de perda de dados e a distância entre os dispositivos.

Os resultados de algumas variáveis importantes como distância, perda de pacote de dados e análise baseada no erro, mostram que o regime de simulação integrada fornece uma solução conjunta para análise de redes de comunicação sem fio, para o controle da $G D$ e para a modelagem da rede elétrica em microrredes inteligentes $(M R I)$, representando a eficácia da solução em rede para o desenvolvimento de uma $M R$.

Diante do cenário apresentado, o objetivo deste trabalho foi desenvolver um ambiente de simulação integrada de microrredes inteligentes de energia para estudar e avaliar o uso de redes de comunicação sem fio para controle da geração distribuída de potência ativa entre as fontes alternativas de geração de energia elétrica e os consumidores de um sistema distribuído em um ambiente de redes inteligentes.

\section{DESCRIÇÃO DA MRI SIMULADA}

As $M R I$ são versões modernas, em pequena escala, do sistema centralizado de eletricidade, que visa atingir metas locais específicas como a redução de $\mathrm{CO}_{2}$, redução de custos e diversificação das matrizes energéticas. As $M R I$, assim como a rede de energia convencional, gera, distribui e regula o fluxo de eletricidade para os consumidores de forma local, sendo uma maneira ideal de integrar recursos renováveis a nível comunitário e permitir a participação do cliente na empresa de eletricidade, formando os blocos de construção do sistema de potência perfeito [7]. Nesta seção são apresentados os 
aspectos metodológicos e materiais para a simulação integrada do circuito elétrico proposto contendo a geração distribuída, o controle da geração de potência e a rede de comunicação sem fio, bem como as ferramentas utilizadas para que o conjunto gerado fosse simulado no ambiente Matlab-Simulink.

O circuito elétrico com o GD é apresentado de forma simplificada na Fig. 1.

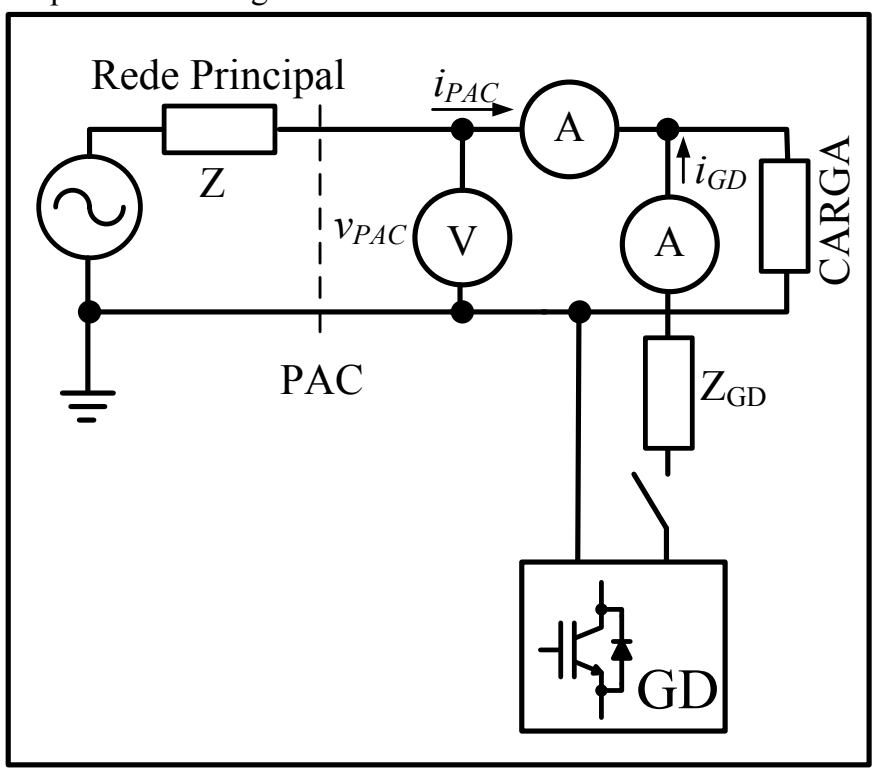

Figura 1: Diagrama do circuito elétrico com o GD.

O circuito da Fig. 1, é composto por uma fonte de tensão monofásica e uma impedância de linha $(Z)$, que se assemelha a uma rede real. A carga é do tipo resistiva com $10 \mathrm{~kW}$ de potência nominal. Os valores respectivos de tensão e corrente da rede e corrente no $G D$, são medidos no ponto de acoplamento comum $(P A C)$, o que permite observar o comportamento da carga. Uma linha tracejada entre a rede principal e a carga com o $G D$ representa o $P A C$. Um $G D$ com capacidade para gerar potência de até $10 \mathrm{~kW}$, foi inserido em paralelo com a carga. No entanto, para analisar o comportamento da $M R$ com o $G D$ e o sistema de controle, o valor de interação foi fixado em $5 \mathrm{~kW}$ e medido pela referência de tensão com a corrente gerada no $P A C$.

O objetivo do $G D$ é gerar potência ativa fundamental $\left(P_{1}\right)$ que, nesse caso, será consumida pela carga, independente da forma de onda da tensão e corrente da fonte.

A Fig. 2 apresenta o controle da geração de potência para o $G D$, onde o erro entre a potência de referência $\left(P_{R e f}\right)$ e a potência medida $\left(P_{m}\right)$, gerada pelo $G D$, passa por um controlador do tipo proporcional integral $\left(P I_{1}\right)$ resultando no valor da potência de saída $\left(P_{S}\right)$ do controlador.

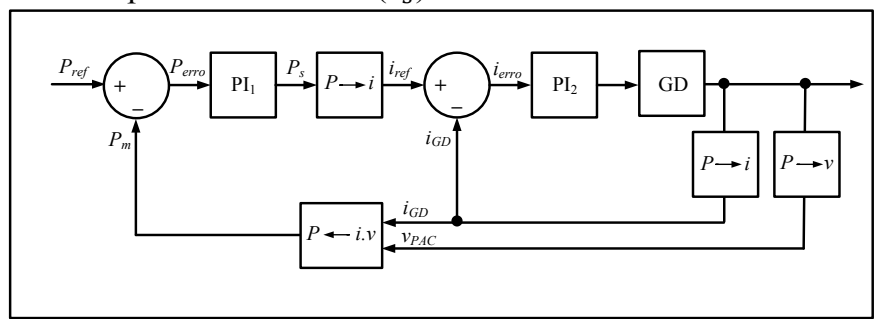

Figura 2: Controle da geração de potência ativa do $G D$.
A potência $P_{m}$ é calculada conforme (1).

$P_{m}=\frac{1}{T} \int_{0}^{T} v_{P A C}^{1} i_{G D}^{1} d t$

na qual: $v_{P A C}^{1}$ e $i_{G D}^{1}$ são respectivamente as parcelas de tensão instantânea fundamental no $P A C$ e corrente instantânea fundamental do $G D$.

A corrente instantânea de referência $i_{R e f}$ para o GD é obtida conforme (2).

$i_{R e f}=\frac{P_{S}}{\left(V_{P A C}^{1}\right)^{2}} v_{P A C}^{1}$

na qual: $V_{P A C}^{1}$ é o valor RMS de $v_{P A C}^{1}$, que pode ser obtido conforme (3).

$V_{P A C}^{1}=\sqrt{\frac{1}{T} \int_{0}^{T}\left(v_{P A C}^{1}\right)^{2} d t}$

Finalmente o erro entre $i_{\text {Ref }}$ e $i_{G D}^{1}$ passa por outro controlador $\mathrm{PI}_{2}$ indo para o $G D$, que nas simulações opera com uma tensão senoidal de saída.

Nessa aplicação, a geração de potência ativa é controlada remotamente através de uma rede de comunicação sem fio. A Fig. 3 apresenta a rede de comunicação proposta, bem como a troca de informações entre cada dispositivo.

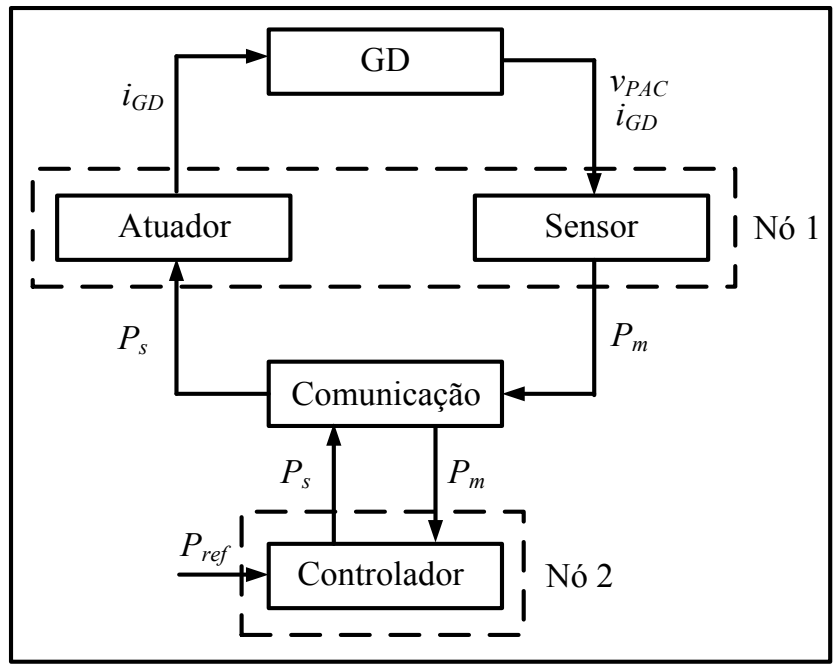

Figura 3: Proposta da rede sem fio.

O dispositivo Nó 1 pode ser um microcontrolador ou microprocessador especializado em processamento digital de sinal $(D S P)$, responsável pelas funções de processar os dados medidos a partir dos sensores de corrente e tensão, para calcular a $P_{m}$, controlar a corrente e também enviar sinais para a comunicação do inversor no $G D$. Nesse caso, o Nó 1 é composto pelo conjunto Sensor-Atuador, onde o sensor recebe os valores de $i_{G D}$ e $v_{P A C}$ e através desses dados calcula $P_{m}$, a qual será enviada via rede sem fio ao $N$ ó 2 , controlador. O Nó 2 é o responsável pelo controle da potência ativa e recebe $P_{\text {ref }}$ e $P_{m}$ vinda do $N$ ó 1 via rede sem fio e envia $P_{s}$ para o Nó 1 via rede sem fío. O Nó 1 recebe por meio do atuador a potência $P_{S}$ e envia ao $G D$ o sinal de $i_{G D}$ a ser injetado na rede. Funções adicionais podem ser direcionadas para o Nó 2, como por exemplo, o controle de supervisão, interface de usuário e comunicação com serviços públicos ou medidores inteligentes. 
Assim, as trocas de informações entre o Nó 1 e o $N$ ó 2 são respectivamente os valores de $P_{m}$ e $P_{s}$, que podem ser transmitidas com uma taxa de amostragem de, pelo menos, a frequência fundamental da rede, ou seja, $60 \mathrm{~Hz}$ possibilitando analisar o comportamento do sistema a cada ciclo. Portanto, para se obter o valor de potência, é necessário ao menos um ciclo de amostragem, já que a frequência da rede elétrica também é de $60 \mathrm{~Hz}$.

A Fig. 4 apresenta o circuito de uma MRI de forma simplificada com as identificações e separações quanto ao tipo de software utilizado no desenvolvimento de cada etapa do circuito.

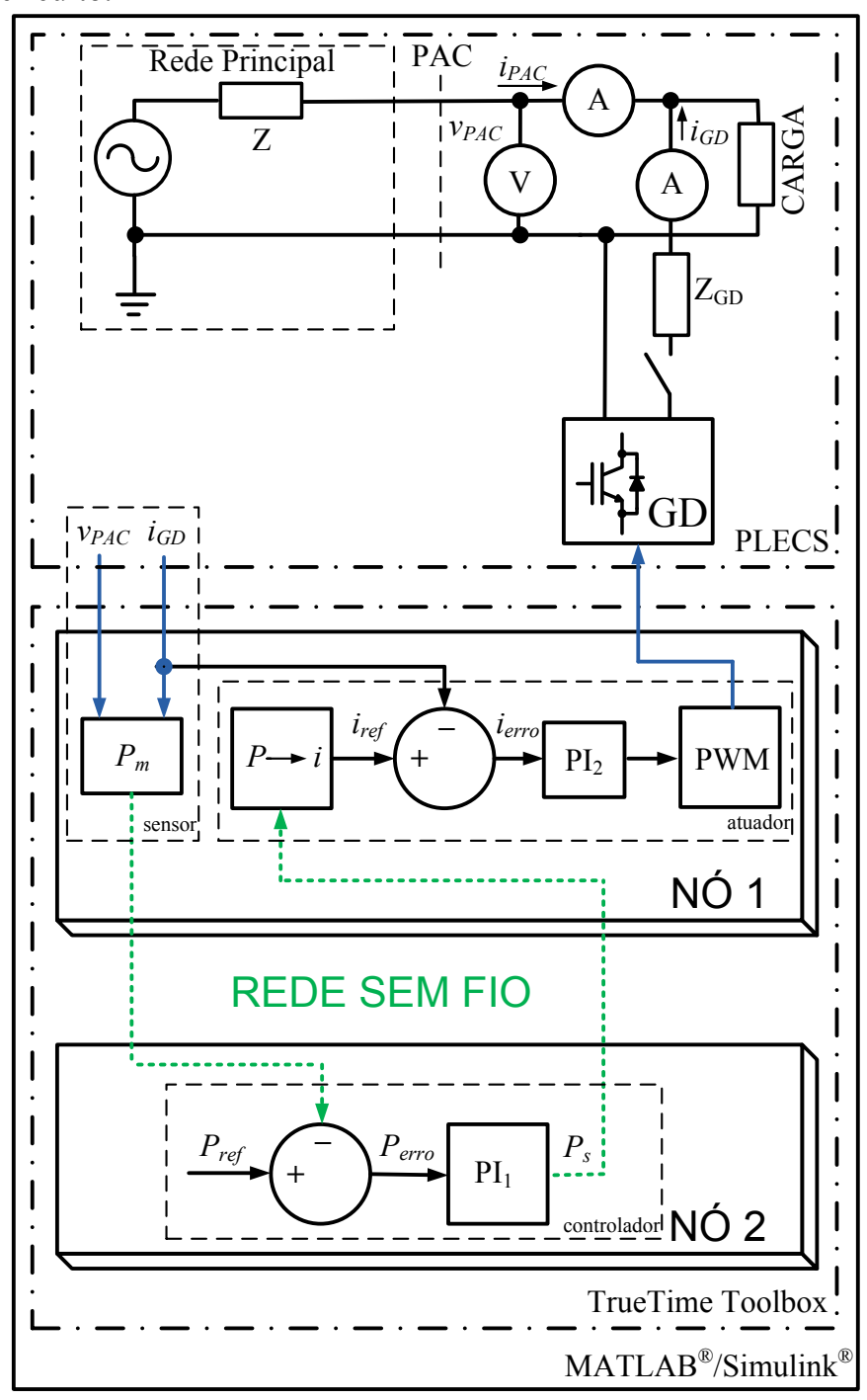

Legenda: $\longrightarrow$ Cabo $\longrightarrow$ Software $\cdots$ Rede sem fio

Figura 4: Circuito de uma $M R I$

\section{MATERIAIS E MÉTODOS}

A rede sem fio foi simulada através do software TrueTime-toolbox no Matlab-Simulink e os blocos são descritos nas próximas subseções.

\section{A. $\quad$ TrueTime Wireless Network}

O bloco de rede sem fio é baseado em eventos e simula o mecanismo de acesso ao meio de comunicação, a transmissão da mensagem na rede e o tempo de processamento, de acordo com o tipo de rede e protocolo escolhido [8].

Cada mensagem transmitida na rede contém informações sobre o dispositivo de origem e de destino da mensagem, dados definidos pelo usuário como as medições realizadas, sinais de controle e tamanho da mensagem. Outas informações específicas sobre características de tempo real como prioridade e deadline das mensagens são opcionais e definidas de acordo com o protocolo escolhido [9].

Esse bloco é usado para representar uma rede sem fio, permitindo acesso ao meio de transmissão e pacote de dados, sendo configurado neste trabalho para os seguintes tipos de protocolos de redes:

- IEEE $802.11 \mathrm{~b} / \mathrm{g}(W L A N)$;

- IEEE 802.15.4 (ZigBee).

A Fig. 5 apresenta o bloco TrueTime Wireless Network.

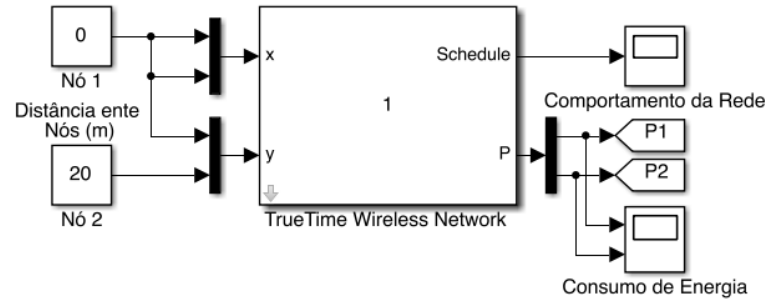

Figura 5: Bloco TrueTime Wireless Network.

onde: Os valores 0 e 20, são constantes que representam a distância entre Nó 1 e $N$ ó 2 que nesse caso foi de 20 metros; Schedule é utilizado para verificar o comportamento da rede durante a transmissão de dados;

$P 1$ e $P 2$ se referem aos consumos de energia na comunicação entre $N$ ó 1 e $N$ ó 2 , respectivamente.

\section{B. $\quad$ TrueTime Kernel}

O bloco de Kernel é composto por uma função do Matlab que simula um computador ou um processador com um Kernel baseado em eventos, operações de conversão $A / D$ e $D / A$, interface de dados $I / O$, comunicação via rede, canal externo para interrupções e variáveis de monitoramento (Schedule e Monitors) [8]. É usado para representar o nó na rede, ou seja, o componente ou dispositivo em que a rede irá efetuar a troca de informações.

Para cada componente ou dispositivo ligado à rede, é necessário a utilização de um bloco desse.

A Fig. 6 apresenta o bloco do TrueTime Kernel do Nó 1 e o bloco bateria utilizado no $N$ ó 1 .

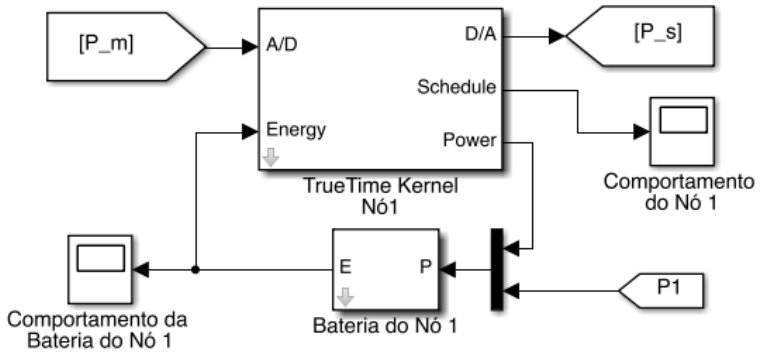

Figura 6: Bloco TrueTime Kernel do Nó 1 e Bloco da Bateria do Nó 1. 
A Fig. 7 apresenta o bloco do TrueTime Kernel do Nó 2 e o bloco bateria utilizado no Nó 2 .

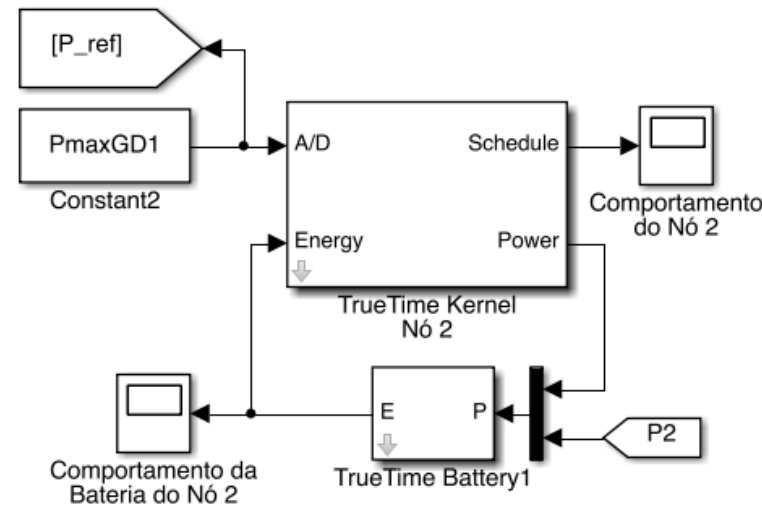

Figura 7: Bloco TrueTime Kernel do Nó 2 e Bloco da Bateria do Nó 2.

no qual: $A / D$ é a entrada de dados analógicos que são convertidos para dados digitais;

Energy é o ponto de conexão que é ligado na conexão $E$ da bateria;

$D / A$ é a saída onde os dados digitais são convertidos para os dados analógicos;

Schedule é o local de verificação dos dados de comportamento do respectivo nó da rede;

Power é o ponto de conexão que é ligado na conexão $P$ da bateria.

\section{TrueTime Battery}

O bloco de bateria foi desenvolvido com o intuito de permitir a simulação de controle via rede em ambiente com robôs móveis, no qual a alimentação dos dispositivos é fornecida por baterias.

A Fig. 6 e Fig. 7 respectivamente, apresentam o bloco TrueTime Battery.

onde: $P$ é o ponto de conexão que é ligado a conexão Power do bloco TrueTime Kernel;

$E$ é o ponto de conexão que é ligado a conexão Energy do bloco TrueTime Kernel.

\section{RESULTADOS E ANÁLISES}

A tensão fornecida pela rede principal é de $127 V_{R M S}$, com uma frequência de $60 \mathrm{~Hz}$. Os valores de impedância da rede são representados por uma equivalente $Z$, onde $R=23,8 m \Omega$ e $L=104 \mu H$. O valor de $Z$ pode ser obtido conforme (4), (5) e (6) respectivamente, ou conforme (7).

$Z=R+j \cdot X$

$X=\omega \cdot L$

$\omega=2 \cdot \pi$

$Z^{2}=R^{2}+X^{2}$

Com o valor da impedância, foi possível calcular o valor do ângulo e da corrente de curto circuito, conforme (8) e (9). $\tan \theta=\frac{X}{R}$

$I_{C C}=\frac{V}{Z}$

O tempo total de simulação foi de 10 segundos e assumindo para esse intervalo de tempo que a potência máxima que pode ser injetada pelo $G D$ foi de $5 \mathrm{~kW}, P_{R e f}$ foi fixado também em $5 \mathrm{~kW}$. Além disso, o $G D$ foi ligado no instante $t=0,1 \mathrm{~s}$ para analisar o comportamento da tensão e corrente instantâneas no $P A C$ e corrente instantânea no $G D$, bem como o comportamento das potências através da comunicação sem fio.

A Fig. 8 apresenta as potências $P_{P A C}, P_{r e f}$ e $P_{m}$.

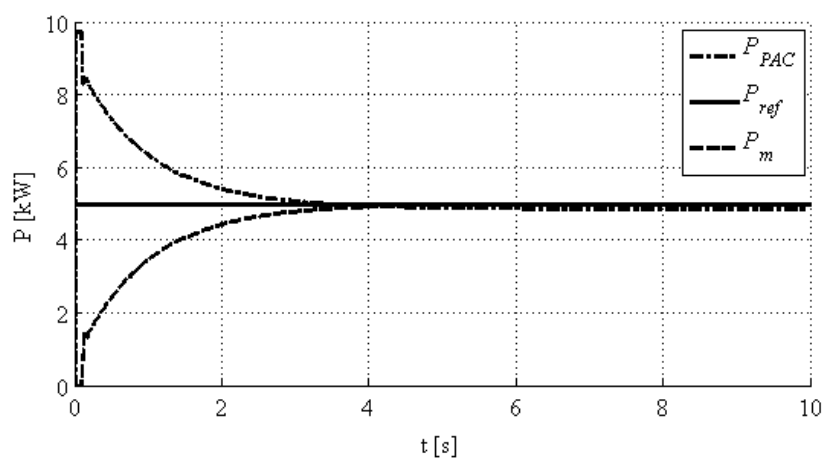

Figura 8: $P_{P A C}, P_{\text {ref }}$ e $P_{m}$.

A Fig. 9 apresenta as formas de onda da $v_{P A C}$ e $i_{P A C}$ no $P A C$ e a Fig. 10 apresenta a corrente $i_{G D}$ nos primeiros segundos de simulação. Nota-se que as correntes estão em fase com a tensão e isso se deve ao fato de que a carga é resistiva.

As oscilações na amplitude das formas de onda de $i_{P A C} \mathrm{e}$ $i_{G D}$, ocorrem devido ao período transitório.

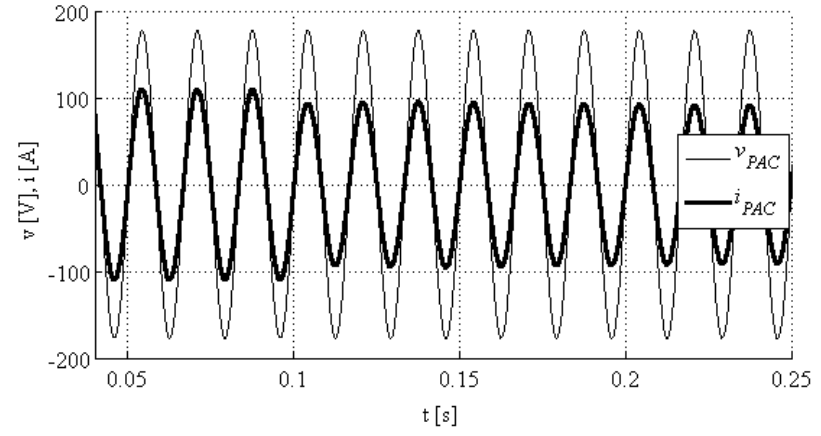

Figura 9: $v_{P A C}, i_{P A C}$.

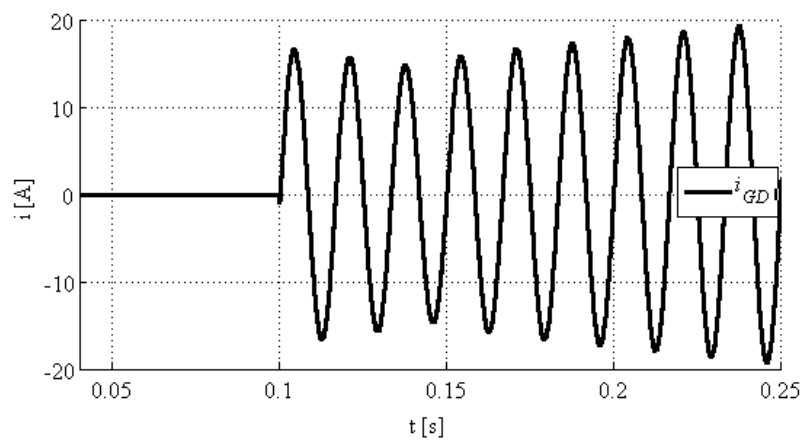

Figura 10: $i_{G D}$. 
As Fig. 9 e Fig. 10 apresentam o comportamento transitório da corrente $i_{G D}$, conforme a entrada em operação do $G D$, demonstrada na Fig. 8.

Foi realizada a análise para dois tipos de protocolos de rede de comunicação sem fio, conforme descritos a seguir:

\section{A. Protocolo IEEE $802.11 \mathrm{~b} / \mathrm{g}(W L A N)$}

Para essa análise, foi considerada uma rede de transmissão de dados ideal, ou seja, com $0 \%$ de probabilidade de perda de dados em toda a sua transmissão.

No circuito da MRI foi utilizado no bloco TrueTime Wireless Network o protocolo de rede IEEE $802.11 \mathrm{~b} / \mathrm{g}$ (WLAN) e configurado conforme [10] a Tabela I.

TABELA I

CONFIGURAÇÃO DO BLOCO TRUETIME WIRELESS NETWORK.

\begin{tabular}{cccc}
\hline \multicolumn{4}{c}{ PARÂMETROS } \\
\hline Item & Descrição & Dados & Unidade \\
\hline $\mathbf{1}$ & Network type & $802.11 \mathrm{~b} / \mathrm{g}$ & - \\
\hline $\mathbf{2}$ & Network Number & 1 & - \\
\hline $\mathbf{3}$ & Number of nodes & 2 & - \\
\hline $\mathbf{4}$ & Data rate & 64000000 & bits $/ \mathrm{s}$ \\
\hline $\mathbf{5}$ & Minimum frame size & 272 & bits \\
\hline $\mathbf{6}$ & Transmit power & 13 & $d b m$ \\
\hline $\mathbf{7}$ & Receiver signal Threshold & -71 & $d b m$ \\
\hline $\mathbf{8}$ & Pathloss function & default & - \\
\hline $\mathbf{9}$ & Pathloss exponent & 3,5 & $1 /$ distance \\
\hline $\mathbf{1 0}$ & ACK timeout & 0,00004 & $\mathrm{~s}$ \\
\hline $\mathbf{1 1}$ & Retry limit & 5 & - \\
\hline $\mathbf{1 2}$ & Error coding threshold & 0,03 & - \\
\hline $\mathbf{1 3}$ & Loss probability (0-1) & 0,0 & - \\
\hline $\mathbf{1 4}$ & Initial seed & 0 & - \\
\hline
\end{tabular}

Além das configurações apresentadas na Tabela I, foram selecionadas as opções:

- Show Schedule output port.

- Show Power consumption output port.

O Nó 1 utiliza o bloco TrueTime Kernel e configurado conforme a Tabela II.

TABELA II

CONFIGURAÇÃO DO BLOCO TRUETIME KERNEL DO NÓ 1

\begin{tabular}{ccc}
\hline \multicolumn{3}{c}{ PARÂMETROS } \\
\hline Item & Descrição & Dados \\
\hline $\mathbf{1}$ & Name of init function (MEX or Matlab) & sensor_atuador_init \\
\hline $\mathbf{2}$ & Init function argument (arbitrary struct) & {[]} \\
\hline $\mathbf{3}$ & Number of analog inputs and outputs & {$[11]$} \\
\hline $\mathbf{4}$ & Number of external triggers & 0 \\
\hline $\mathbf{5}$ & (Network and) Node Number(s) & 1 \\
\hline $\mathbf{6}$ & Local clock offset and drift & {$[00]$} \\
\hline
\end{tabular}

A Tabela III apresenta os parâmetros de configuração do bloco do Nó 2 .

Além das configurações apresentadas nas Tabelas II e III respectivamente, foram selecionadas as opções:
- Show Schedule output port.

- Show Energy supply input port.

- Show Power consumption output port.

TABELA III

CONFIGURAÇÃO DO BLOCO TRUETIME KERNEL DO NÓ 2.

\begin{tabular}{ccc}
\hline \multicolumn{3}{c}{ PARÂMETROS } \\
\hline Item & Descrição & Dados \\
\hline $\mathbf{1}$ & Name of init function (MEX or Matlab) & controlador_init \\
\hline $\mathbf{2}$ & Init function argument (arbitrary struct) & {[]} \\
\hline $\mathbf{3}$ & Number of analog inputs and outputs & {$[1$ 0] } \\
\hline $\mathbf{4}$ & Number of external triggers & 0 \\
\hline $\mathbf{5}$ & (Network and) Node Number(s) & 2 \\
\hline $\mathbf{6}$ & Local clock offset and drift & {$[00]$} \\
\hline
\end{tabular}

O parâmetro de configuração do bloco TrueTime Battery do Nó 1 e Nó 2 respectivamente se refere ao campo Initial energy, que apesar de não apresentar unidade de medida no bloco, é em Joule, cujo valor inserido foi de 2694,6 conforme [4].

O valor da alimentação da bateria foi baseado em uma fonte de energia real composta por três pilhas recarregáveis tipo $A A$ de Níquel Metal Hidreto- NiMH, cuja tensão é de $1,2 \mathrm{~V}$ cada, com uma carga nominal de $2300 \mathrm{mAh}$ com alta capacidade de corrente e considerada descarregada quando sua tensão for igual a $1 V$ [11].

As pilhas ligadas em série, totalizam uma tensão de $3,6 \mathrm{~V}$ já que o módulo da rede $W L A N$ funciona entre $3,14 \mathrm{~V}$ a $3,46 V[12]$.

Os dados de energia da bateria podem ser obtidos conforme (10).

$P_{n}=\frac{m A h \cdot V_{C C}}{1000}$

onde $P_{n}$ é a potência nominal em horas cuja unidade é em $W h$.

Para transformar Wh em Joules, para introduzir o valor correto no campo Initial energy da bateria do bloco do TrueTime Battery, tem-se (11).

$E=W \cdot s$

A Fig. 11 apresenta detalhes do uso da rede sem fio com os seus respectivos nós na $M R I$ durante a simulação.

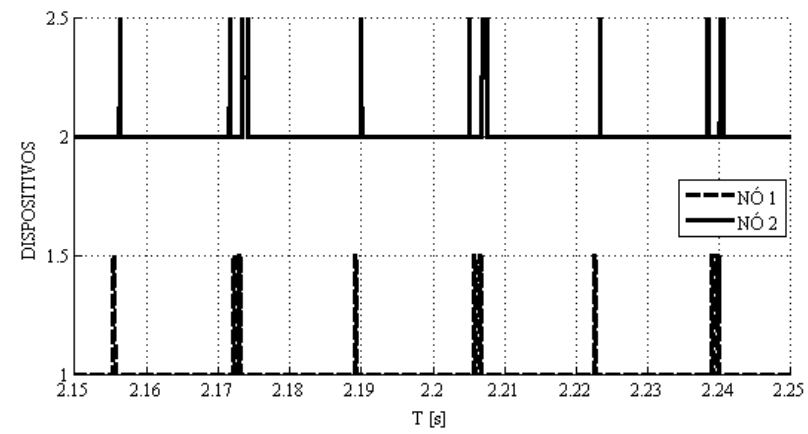

Figura 11: Detalhes no uso da rede sem fio com os Nós na MRI (Nó 1-Sensor / Atuador e Nó 2-Controlador).

A Tabela IV apresenta o processamento da rede, do Nó 1 e do Nó 2 respectivamente no que diz respeito a enviar ou 
receber dados e teste de comunicação, em porcentagem dividido em três tempos, ou seja:

- $\quad t_{O N}-$ Tempo em que a rede está transmitindo.

- $t_{O F F}-$ Tempo em que a rede não está transmitindo.

- $t_{W A I T}-$ Tempo em que a rede está aguardando um nó prioritário terminar de transmitir para que o mesmo retorne a transmissão que havia sido iniciada.

Pode-se observar que toda a transmissão de dados na rede permaneceu inativa a maior parte do tempo de simulação.

Essa informação é importante devido ao fato de que o consumo dos dispositivos estão estritamente relacionados com o $t_{O N}$ juntamente com o $t_{\text {WAIT }}$ das tarefas.

TABELA IV

RESULTADO DA SIMULAÇÃO INTEGRADA DA MRI COM $20 \mathrm{~m}$ DE DISTÂNCIA E SEM PERDA DE DADOS NA TRANSMISSÃO (WLAN)

\begin{tabular}{cccc}
\hline & $\begin{array}{c}\text { Rede sem fio } \\
\text { Uso (\%) }\end{array}$ & $\begin{array}{c}\text { Nó } 1 \\
\text { Uso (\%) }\end{array}$ & $\begin{array}{c}\text { Nó } 2 \\
\text { Uso (\%) }\end{array}$ \\
\hline $\boldsymbol{t}_{\boldsymbol{O N}}$ & 1,65 & 12,75 & 5,04 \\
\hline $\boldsymbol{t}_{\boldsymbol{O F F}}$ & 91,86 & 79,00 & 94,17 \\
\hline $\boldsymbol{t}_{\boldsymbol{W A I T}}$ & 6,50 & 8,25 & 0,80 \\
\hline
\end{tabular}

\section{B. Protocolo IEEE 802.15.4 (ZigBee)}

Para essa análise, foi considerada uma rede de transmissão de dados ideal, ou seja, com $0 \%$ de probabilidade de perda de dados em toda a sua transmissão.

No circuito de $M R I$ proposto foi utilizado no bloco TrueTime Wireless Network o protocolo de rede IEEE 802.15.4 (ZigBee) e configurado conforme a tabela V. Devido ao tipo de protocolo, foram mudadas apenas a linha 1 que se refere ao tipo de rede, a linha 4 que se refere ao tamanho da mensagem a ser transmitida, a linha 5 que se refere à transmissão de energia e a linha 6 que se refere a sensitividade quanto ao recebimento da informação, conforme [12].

TABELA V

CONFIGURAÇÃO DO BLOCO TRUETIME WIRELESS NETWORK.

\begin{tabular}{cccc}
\hline \multicolumn{4}{c}{ PARÂMETROS } \\
\hline Item & Descrição & Dados & Unidade \\
\hline $\mathbf{1}$ & Network type & 802.15 .4 & - \\
\hline $\mathbf{2}$ & Network Number & 1 & - \\
\hline $\mathbf{3}$ & Number of nodes & 2 & - \\
\hline $\mathbf{4}$ & Data rate & 250000 & bits/s \\
\hline $\mathbf{5}$ & Minimum frame size & 272 & bits \\
\hline $\mathbf{6}$ & Transmit power & 18 & dbm \\
\hline $\mathbf{7}$ & Receiver signal Threshold & -101 & dbm \\
\hline $\mathbf{8}$ & Pathloss function & default & - \\
\hline $\mathbf{9}$ & Pathloss exponent & 3,5 & $1 /$ distance \\
\hline $\mathbf{1 0}$ & ACK timeout & 0,00004 & s \\
\hline $\mathbf{1 1}$ & Retry limit & 5 & - \\
\hline $\mathbf{1 2}$ & Error coding threshold & 0,03 & - \\
\hline $\mathbf{1 3}$ & Loss probability (0-1) & 0,0 & - \\
\hline $\mathbf{1 4}$ & Initial seed & 0 & - \\
\hline & & &
\end{tabular}

As configurações dos blocos de Nó 1 e $N$ ó 2, não tiveram alterações, permanecendo com os valores conforme as Tabelas
II e III.

O parâmetro de configuração do bloco TrueTime Battery do Nó 1 e Nó 2 respectivamente se refere ao campo Initial energy, que apesar de não apresentar unidade de medida no bloco, é em Joule, cujo valor inserido foi de 4968, conforme [4].

As pilhas ligadas em série, totalizam uma tensão de $3,6 \mathrm{~V}$ já que o módulo da rede $\mathrm{ZigBee}$ funciona entre $2,7 \mathrm{~V}$ a $3,6 \mathrm{~V}$ [12].

Os dados de energia da bateria podem ser obtidos conforme (10), (11) e (12).

A Fig. 12 apresenta detalhes do uso da rede sem fio com os seus respectivos nós na $M R I$ durante a simulação.

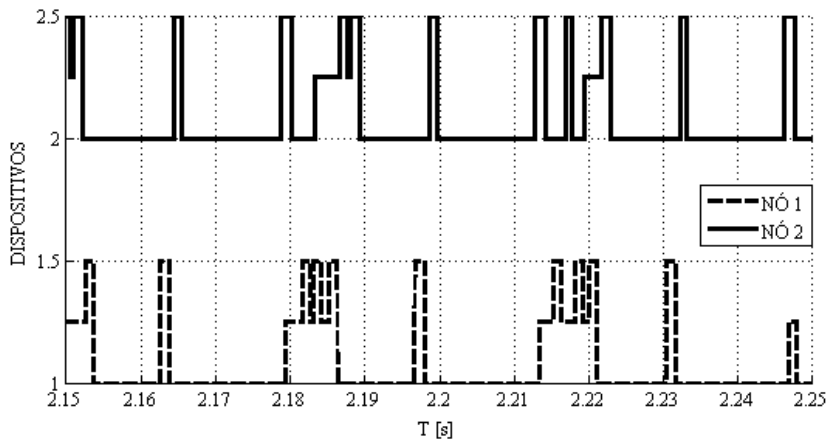

Figura 12: Detalhes no uso da rede sem fio com os nós na MRI (Nó 1-Sensor / Atuador e Nó 2-Controlador).

A Tabela VI apresenta o processamento da rede, do Nó 1 e do Nó 2 respectivamente no que diz respeito a enviar ou receber dados e teste de comunicação.

TABELA VI

RESULTADO DA SIMULAÇÃO INTEGRADA DA MRI COM $20 \mathrm{~m}$ DE DISTÂNCIA E SEM PERDA DE DADOS NA TRANSMISSÃO (ZigBee).

\begin{tabular}{cccc}
\hline & $\begin{array}{c}\text { Rede sem fio } \\
\text { Uso (\%) }\end{array}$ & $\begin{array}{c}\text { Nó } 1 \\
\text { Uso (\%) }\end{array}$ & $\begin{array}{c}\text { Nó } 2 \\
\text { Uso (\%) }\end{array}$ \\
\hline $\boldsymbol{t}_{\boldsymbol{O N}}$ & 27,12 & 11,35 & 3,77 \\
\hline $\boldsymbol{t}_{\boldsymbol{O F F}}$ & 48,03 & 84,33 & 96,23 \\
\hline $\boldsymbol{t}_{\boldsymbol{W A I T}}$ & 24,85 & 4,32 & 0 \\
\hline
\end{tabular}

Foi analisado o desempenho do controle através de ITAE e ISE conforme [13].

A. ITAE

O valor da Integral do Tempo multiplicado pelo valor Absoluto do Erro-ITAE do termo em inglês Integral of Time multiplied by the Absolute Error, é um dos indicadores de desempenho mais utilizados para avaliar o desempenho de controle [13]. Baseia-se no erro da variável controlada, por exemplo, a diferença entre o valor atual da variável do processo e a amplitude do sinal de controle.

O valor de ITAE é obtido conforme (13) e é indicada para reduzir a contribuição do valor de desempenho.

$\operatorname{ITAE}=\int_{0}^{T} t|e(t)| d t$

O ITAE é indicado para reduzir a contribuição de grandes erros iniciais no valor da integral de desempenho, bem como 
enfatizar os erros que acontecem mais tarde na resposta.

A Fig. 13 apresenta a análise de desempenho baseada no erro, em relação a Fig. 8, referente a diferença entre $P_{\text {ref }}$ constante no $N$ ó 2 e $P_{m}$ no sensor do Nó 1 enviada via rede sem fio WLAN para o Nó 2 .

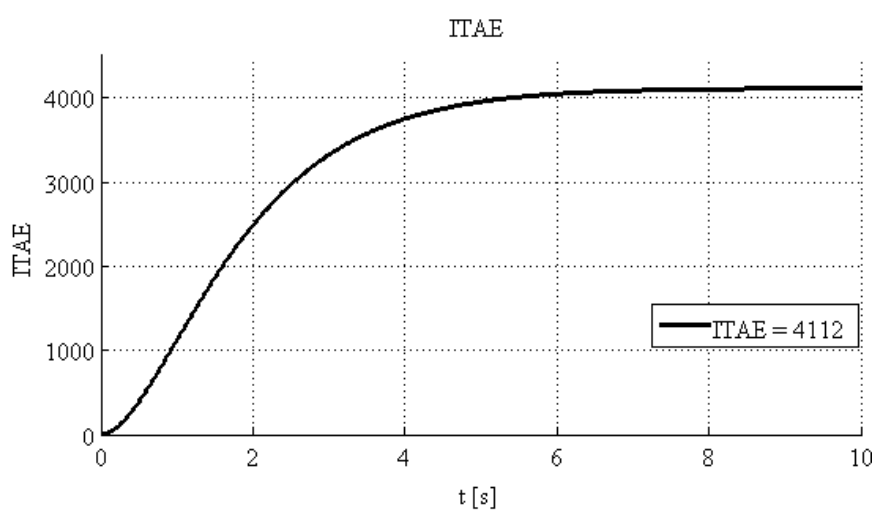

Figura 13: Análise de desempenho baseada no erro entre $P_{r e f}$ e $P_{m}$ através de ITAE.

A Fig. 14 apresenta a análise de desempenho baseada no erro, em relação a Fig. 8, referente a diferença entre $P_{\text {ref }}$ constante no Nó 2 e $P_{m}$ no sensor do $N$ ó 1 enviada via rede sem fio ZigBee para o Nó 2.

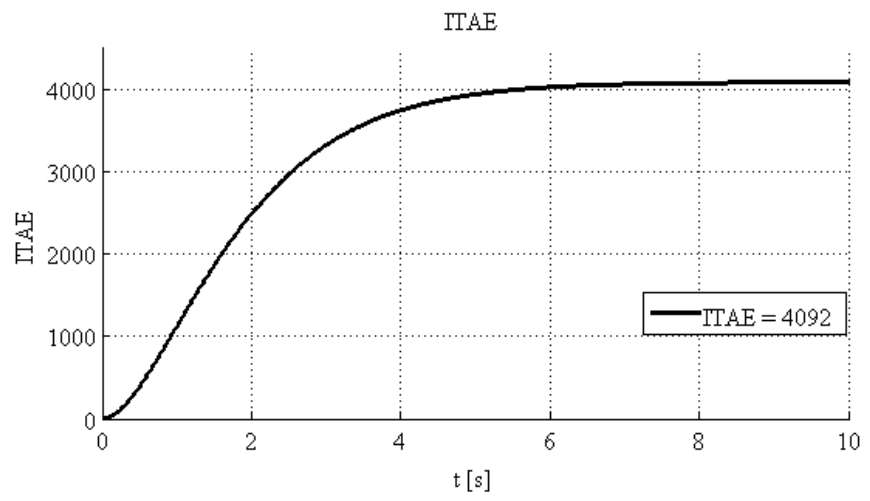

Figura 14: Análise de desempenho baseada no erro entre $P_{r e f}$ e $P_{m}$ através de ITAE.

\section{B. ISE}

Outro índice de desempenho no erro, muito utilizado também é a Integral do Quadrado do Erro-ISE do termo em inglês Integral Square of the Error. Essa análise é utilizada para discriminar sistemas excessivamente superamortecidos dos subamortecidos, ou seja, dá grande peso aos erros grandes e pequenos pesos aos erros pequenos e, devido a isso, não é recomendado para avaliar sistemas somente com erros pequenos [13].

O limite superior é um valor obtido de modo que a integral tenda a um valor estacionário.

O valor de ISE é obtido conforme (14).

$\mathrm{ISE}=\int_{0}^{T} e^{2}(t) d t$

A Fig. 15 apresenta a análise de desempenho baseada no erro, referente ao comportamento do processamento do uso da rede $W L A N$ em relação a $P_{\text {ref }}$ constante no $N$ ó 2 e a $P_{m}$ no sensor do Nó 1 enviada via rede sem fio para o $N o ́ 2$, enquanto que o comportamento do processamento do uso da rede ZigBee foi idêntico inclusive quanto ao valor.

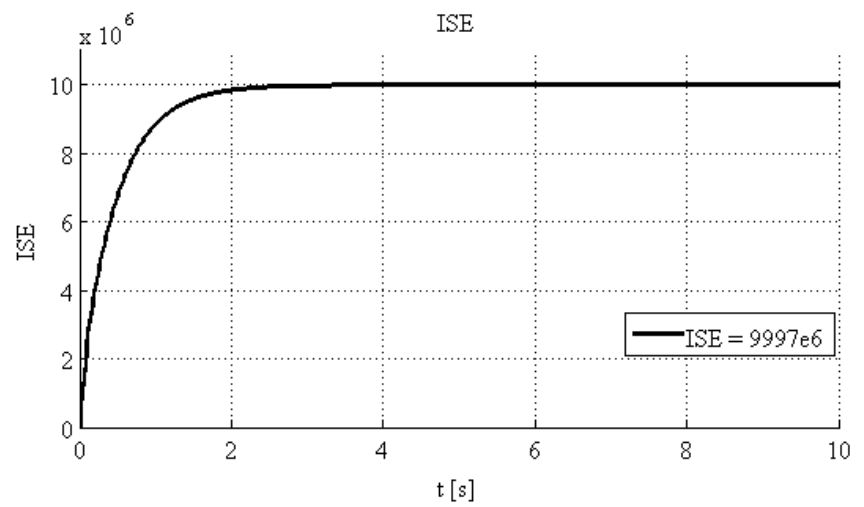

Figura 15: Análise de desempenho baseada no erro entre $P_{r e f}$ e $P_{m}$ através de ITAE.

Através dos dados apresentados, foi possível observar que a porcentagem do uso da rede ZigBee é bem maior, se comparado com a rede $W L A N$, porém, o tempo de espera da rede ZigBee também é bem maior quando comparado com a rede $W L A N$, consequentemente, o tempo em que a rede ZigBee fica em off é de 48,03\% enquanto que o mesmo da rede $W L A N$ é de $91,86 \%$. Através desses valores, nesta configuração é possível estimar que na rede ZigBee seria possível ter no máximo mais dois $G D$ no mesmo circuito enquanto que na rede Wifi seria possível estimar muito mais $G D$. Isso se deve as características específicas de cada tipo de rede, como mostra as Tabelas I e V respectivamente. Nestas configurações, é possível observar que a porcentagem do uso do processador do Nó 1 em ambas as redes apresentam valores bem próximos, bem como os valores do uso do processador do Nó 2 .

Os gráficos de análise de desempenho baseada no erro, bem como os valores obtidos, demonstram que esta configuração adotada para a simulação da $M G I$, permite a utilização dos dois tipos de rede, ou seja, WLAN ou ZigBee, pois são efetivas e viáveis em se tratando desta análise.

\section{CONCLUSÃO}

Este trabalho apresentou estudos da comunicação entre dispositivos de geração distribuída em um ambiente de microrredes inteligentes. O ambiente de simulação integrada desenvolvido para $M R I$ incorporou os pontos fortes de cada ferramenta no mesmo ambiente de desenvolvimento. $\mathrm{O}$ software PLECS-Blockset foi usado para modelagem elétrica, o software TrueTime-toolbox para comunicação e análise em tempo real e ambos em ambiente Matlab-Simulink onde foi realizado o projeto de controle. A flexibilidade e a quantidade de parâmetros de saída podem ser obtidas com o esquema de simulação integrada representam um diferencial na solução. Para um cenário de $M R$ assumindo um $G D$ sendo o controlador distribuído, um nó da rede foi responsável pelo controle do inversor e as medições (Nó 1), enquanto que o segundo nó foi responsável pelo controle da potência ativa 
(Nó 2). A divisão do controle entre dois dispositivos permite a possibilidade de acessar remotamente e controlar a geração de potência ativa usando uma $M R I$ em que fornecedores e utilitários seriam ligados e interagissem. Ao usar o software TrueTime-toolbox, foi possível modelar a rede de comunicação no mesmo ambiente em que o controle e o circuito elétrico foram modelados. Além disso, algumas variáveis importantes sobre a troca de dados, como o período de tempo em que cada transmissão de mensagem era ativa ou inativa e a descarga da bateria, foram simulados e discutidos. O desempenho global da comunicação usando a rede WLAN e ZigBee foram avaliadas sob diferentes dispositivos. Para a $M R$ estudada neste trabalho, ambos os parâmetros tiveram um pequeno impacto na operação de $M R I$. Foi possível estimar que a rede do tipo ZigBee comporta no máximo mais dois $G D$ enquanto que a rede $W L A N$ comporta muito mais, já que, devido as características específicas da rede, a mesma utiliza uma porcentagem de tempo de uso da rede, bem menor em comparação à rede ZigBee. Finalmente, este estudo preliminar é um ponto de partida para uma espécie de outros estudos sobre a modelagem da rede de comunicação para $M R I$, como a influência da distância entre os nós, a perda de pacotes durante a transmissão dos dados e a frequência de troca de dados sobre o controle da geração de potência ativa e a adição de outros nós na rede.

\section{AGRADECIMENTOS}

Os autores agradecem ao Grupo de Automação e Sistemas Integráveis $(G A S I)$ da UNESP - Sorocaba pelo apoio dado durante o desenvolvimento deste projeto.

\section{REFERÊNCIAS}

[1] EL-HAWARY, Mohamed E. The Smart Grid - State-of-the-art and future trends. Electric Power Components and Systems, v. 42, n. 3 - 4, p. $239-$ 250,2014

[2] VB - Venturebeat Interpreting Innovation. How secure is the smart grid? $<$ http://venturebeat.com/2011/02/01/how-secure-is-the-smart-grid/> Acesso em Janeiro 2016.

[3] PAREDES, H. K. M. Teoria da Potência Conservativa: Uma nova Abordagem para o Controle Cooperativo de Condicionadores de Energia e Considerações sobre Atribuição de Responsabilidades. 2011. 257 f. Tese (Doutorado) - Curso de Engenharia Elétrica, Universidade Estadual de Campinas, Campinas, 2011.

[4] CERVIN, Anton; HENRIKSSON, Dan; OHLIN, Martin. TRUETUME 2.0 beta - Reference Manual. Department of Automatic Control, Lund University (June 2010), 2010. Disponível em: $<$ http://www.control.lth.se/attic/truetime/report-2.0-beta5.pdf>. Acesso em: Novembro. 2015.

[5] MAJUMDER, Ritwik; BAG, Gargi; CHAKRABARTI, Saikat Performance of Power Electronic Interfaced DERs Integrated with Communication Network. In: 2011 IEEE Power and Energy Society General Meeting. IEEE, 2011. p. $1-7$.

[6] PLECS - The Simulation Platform for Power Electronic Systems - User Manual - Version 4.0. Disponível em: <http://www.plexim.com/plecs>. Acesso em: 26 de fevereiro de 2016.

[7] GALVIN, BOB. (2012). Disponível em: $<$ http://www.galvinpower.org/microgrids $>$. Acesso em: Novembro. 2015.

[8] CERVIN, Anton; OHLIN, Martin; HENRIKSSON, Dan. Simulation of Networked Control Systems Using TrueTime. In: 3rd International Workshop on Networked Control Systems: Tolerant to Faults. 2007.

[9] GODOY, Eduardo Paciência. Desenvolvimento de Sistemas de Controle via Rede (NCS) para Aplicações em Redes com Protocolo CAN. Tese de Doutorado. Universidade de São Paulo, São Carlos, 2011.

[10] DIGI (2016). XBEE WI-FI. Embedded Wi-Fi modules provide ultra lowpower $802.11 \mathrm{~b} / \mathrm{g} / \mathrm{n}$ communications in the flexible XBee hardware and software footprint. Disponível em: https://www.digi.com/pdf/ds_xbeewifis6b.pdf. Acesso em: Setembro, 2016.

[11] THEZOLIN1, ANDRÉ TORTORELLI et al. PILHAS RECARREGÁVEIS: ANÁLISE DE SUAS VANTAGENS E DESVANTAGENS EM RELAÇÃO ÀS NÃO RECARREGÁVEIS. 2010.

[12] DIGI (2016). XBEE ZIGBEE. Embedded ZigBee modules provide OEMs with a simple way to integrate mesh technology into their application. Disponível em: https://www.digi.com/pdf/ds_xbee_zigbee.pdf. Acesso em: Setembro, 2016.

[13] GODOY, Eduardo Paciência. Critérios de Desempenho de Sistemas de Controle - Tipos e Modificações de Controladores PID Industriais. v. 1, 2014.

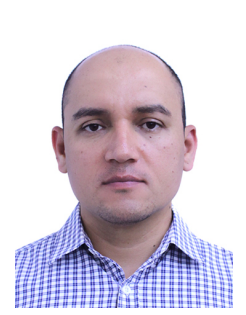

Jefferson Aparecido Dias nasceu em Itu-SP - Brasil, em 1979. Bacharel em Engenharia Mecatrônica pelo CEUNSP - Centro Universitário Nossa Senhora do Patrocínio, na cidade de Salto - SP, Brasil, em 2011. Especialista em Automação Industrial formado pelo SENAI - Serviço Nacional de Aprendizagem Industrial, na cidade de São Caetano do Sul - SP, Brasil em 2014. Mestre em Engenharia Elétrica pela UNESP - Universidade Estadual Paulista, Bauru - SP Brasil, em 2016. Aluno do curso de Doutorado Acadêmico do Programa de Pós-graduação em Engenharia Elétrica da UNESP - Universidade Estadual Paulista, Bauru - SP, Brasil. Atualmente é professor substituto na Faculdade de Engenharia de Controle e Automação da UNESP, Sorocaba - SP, Brasil e professor da FACENS - Faculdade de Engenharia de Sorocaba - SP, Brasil. Sua área de pesquisa está relacionada ao estudo da comunicação entre os dispositivos de medição em um ambiente de redes inteligentes. É membro da ISA e da SBA.

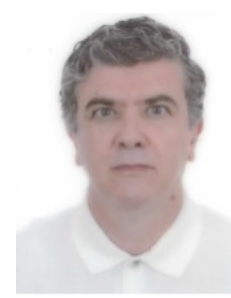

Paulo José Amaral Serni, nasceu em Botucatu - SP Brasil, em 1957. Mestre (1992) e Doutor (1999) em Engenharia Elétrica pela Faculdade de Engenharia Elétrica da UNICAMP, Campinas - SP, Brasil. De 1987 a 2012 foi Professor da Faculdade de Engenharia Elétrica da UNESP, Bauru - SP, Brasil. Desde 2012 é Professor Assistente da Faculdade de Engenharia de Controle e Automação da UNESP, Sorocaba - SP, Brasil. Suas áreas de interesse são: eletrônica de potência, máquinas elétricas e modelos eletromagnéticos dinâmicos, qualidade de energia e redes inteligentes, instrumentação e sistemas de medição.

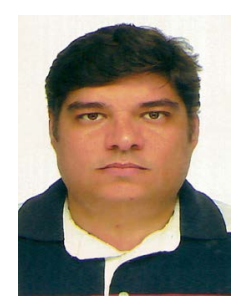

Átila Madureira Bueno nasceu em Santo André - SP, Brasil, em 1973. Bacharel (1999) em Engenharia da Computação pela Universidade Brás Cubas, Brasil. Mestre (2004) em Engenharia Espacial pelo Instituto Nacional de Pesquisas Espaciais - INPE, Brasil e Doutor (2009) em Engenharia Elétrica na área de Engenharia de Sistemas, pela Escola Politécnica da Universidade de São Paulo - USP, Brasil. Atualmente é Professor na Universidade Estadual Paulista - UNESP, Sorocaba - SP, Brasil. Suas áreas de interesse incluem: sistemas de controle, sincronização e sistemas de controle não-lineares.

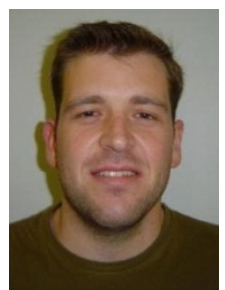

Eduardo Paciência Godoy é Bacharel em Engenharia de Controle e Automação pela UNIFEI - Universidade Federal de Itajubá, na cidade de Itajubá - MG, Brasil Mestre (2007) e Doutor (2011) em Engenharia Mecânica na USP - Universidade de São Paulo, na cidade de São Carlos - SP, Brasil. Atualmente é Professor Adjunto da Faculdade de Engenharia de Controle e Automação da UNESP - Universidade Estadual Paulista, Sorocaba SP, Brasil. Suas áreas de interesse são de pesquisas que incluem redes industriais e automação, sistemas de controle via rede (NCS), redes sem fio e de telemetria, eletrônica embarcada e internet das coisas. 